Проняева Л.И. Павлова А.В.

\section{РАЗВИТИЕ СИСТЕМЫ МЕЖОРГАНИЗАЦИОННОГО УПРАВЛЕНИЯ В КЛАСТЕРАХ}

1) доктор экономических наук, профессор. Российская академия народного хозяйства и государственной службы при Президенте Российской Федерации, Орловский филиал. б. Победы 5а, Орёл, 302028, Российская Федерация

Электронный адрес: pli.dom@mail.ru

2) аспирант кафедры «Экономика и экономическая безопасность». Российская академия народного хозяйства и государственной службы при Президенте Российской Федерации, Орловский филиал. б. Победы 5a, Орёл, 302028,

Российская Федерация. Электронный адрес:anna-pavlova1010@yandex.ru

Аннотация. В статье раскрывается сущность понятий партнерских отношений, межорганизационного управления и его принципов. Дана оценка кластерной формы объединения организаций, являющейся одним из инструментов повышения их конкурентоспособности. Проведено исследование форм управления в кластерных объединениях и предложены рекомендации по совершенствованию механизма межорганизационного управления. В статье затронуты проблемы сетевого взаимодействия организаций, создания кластерных структур, развития интеграционных связей, послужившие основной для определения состава принципов развития межорганизационных отношений и управления ими. Произведена авторская группировка и систематизация взаимосвязанных принципов управления межорганизационными отношениями в кластерах на основе выделения стадий жизненного цикла организаций и видов управления: стадия формирования кластерных структур; стадия функционирования кластерных объединений; принципы стратегического управления кластерными структурами.

Ключевые слова: кластерные объединения; межорганизационное управление; механизм управления; формы и принципы управления.

Pronyaeva L.I.

Pavlova A.V.

\title{
DEVELOPMENT OF THE INTERORGANIZATIONAL MANAGEMENT
} SYSTEM IN CLUSTERS

1) Doctor of Economics, Professor. The Russian Presidential Academy of National Economy and Public Administration, the Orel Branch. 5a Pobedy Blvd., Orel, 302028, Russian Federation. E-mail: pli.dom@mail.ru

2) Postgraduate Student, Department of Economics and Economic Security. The Russian Presidential Academy of National Economy and Public Administration, the Orel Branch. 5a Pobedy Blvd., Orel, 302028, Russian Federation

E-mail: anna-pavlova1010@yandex.ru

\begin{abstract}
The article reveals the essence of the concepts of partner relations, interorganizational management and its principles. The cluster forms of an organizational association, being one of the tools increasing their competitiveness, have been estimated herein. A research of the forms of management in cluster associations has been carried out and some recommendations of how to improve a mechanism of interorganizational management have been offered. The article covers the problems of network interaction between the organizations, the formation of cluster structures, and the development of integration communications, which served as a basis for outlining the structure of principles for the development of interorganizational relations and how to manage them. The author conducted the grouping and systematization of interconnected principles of how to manage the interorganizational relations in clusters on the basis of allocating the stages of the organizations' life cycle and the types of management: the stage, at which the cluster structures are formed; the stage, at which the cluster association functions; the principles of strategic management of cluster structures.
\end{abstract}

Keywords: cluster associations; interorganizational management; a controlling mechanism; forms and principles of management 
Актуальность. В условиях глобализации и интеграции экономических систем субъекты хозяйствования ориентированы на создание и развитие конкурентных преимуществ в целях повышения эффективности деятельности. На современном этапе развития экономики конкуренция определяется не только доступом к ресурсам и рынкам сбыта, но и доступом к информации и партнерам по бизнесу, таким образом, наличие устойчивых партнерских взаимосвязей является конкурентным преимуществом организации.

Важным аспектом является качество управления партнерскими отношениями. Отечественные предприятия, ориентированные на межфирменное партнерство часто сталкиваются с проблемой эффективного управления межорганизационными отношениями. В связи с этим, актуальным является проблема исследования вопросов межорганизационного управления.

В настоящее время одной из форм межфирменной интеграции и кооперации является кластерное объединение организаций. Реализация кластерной политики представляет собой один из механизмов повышения конкурентоспособности. Необходимо отметить, что кластерная концепция может быть успешно использована для стимулирования экономического развития на разных политико-географических уровнях: на международном уровне; на федеральном уровне; на региональном уровне; на уровне города и для экономически и социально депрессивных районов больших городов [8].

Для нашей страны кластерный подход является новым инструментом экономического развития и для того, чтобы кластерные формы организации стали эффективным механизмом повышения конкурентоспособности необходимо комплексное изучение вопросов развития системы управления межорганизационными отношениями.

Изложение основного материала. В условиях современного рынка любое предприятие нуждается в эффективном и быстром взаимодействии с экономической средой. Такое межорганизационное взаимодействие осуществляется путем установления партнерских связей.

По мнению А. Уилсона и К. Чарлтона, партнерские связи представляют собой «совместную работу двух и более организаций по использованию своих различных ресурсов в целях достижения результатов, недоступных этим организациям по отдельности» [12].

На взгляд К.Ф. Грея, «партнерство - это нечто большее, чем набор целей и процедур; это состояние ума, философия, как вести дела с другими организациями. Партнерство представляет обязательства всех участников проекта уважать, доверять и сотрудничать» [1].

По нашему мнению, партнерские отношения это взаимовыгодные экономические отношения субъектов рынка, устанавливаемые с целью повышения конкурентоспособности и эффективности деятельности на основе сотрудничества.

Кластерные формы объединения организаций играют важную роль в качестве фактора экономического роста и являются одним из инструментов повышения конкурентоспособности. Согласно Концепции долгосрочного социальноэкономического развития РФ на период до 2020 года, предусмотрено создание «сети территориально-производственных кластеров, реализующих конкурентный потенциал территорий». Таким образом, кластерный подход является приоритетным направлением экономической политики России.

M. Портер отмечает функции, которые выполняют кластеры в экономическом развитии любой страны:

1) Кластеры - критические двигатели в экономической структуре национальной и региональной экономики. Процветание региона зависит от значимых позиций в определенном количестве конкурентоспособных кластеров.

2) Кластеры могут определять фундаментальные задачи в национальных или региональных условиях ведения бизнеса: кластеры в большой мере соотносятся с природой конкуренции и микроэкономическими факторами, которые влияют на конкурентные преимущества.

3) Кластеры обеспечивают новый способ мышления в сфере экономики и усилий по развитию ее организации. Так, кластер заставляет пересмотреть роли частного сектора, правительства, торговых ассоциаций, образовательных и исследовательских учреждений в экономическом развитии, а также определить общие возможности, а не только общие проблемы фирм и компаний всех форм собственности [11].

Оценивая перспективы развития российской экономики, М. Портером, выделены факторы, препятствующие кластеризации в России:

- недостаточная развитость инфраструктуры;

- сильный административный ресурс, проявляющийся в высокой степени вмешательства государства в частный сектор экономики;

- слабая судебная система;

- несовершенство нормативной базы, создающей барьеры для экономического развития; 
- высокая сырьевая зависимость экономики, отвечающая политическим интересам и обеспечивающая национальную безопасность;

- наличие компаний-гигантов, занимающих ключевое место в экономике, неразвитая конкуренция [13].

Несмотря на широкое освещение отечественными и зарубежными учеными вопросов управления кластерными структурами, в экономической литературе отсутствует четкое определения категорий межорганизационного управления, его форм и принципов управления кластерными объединениями организаций.

По нашему мнению, межорганизационное управление - это совокупность мероприятий, методов и инструментов, позволяющих формировать, координировать и контролировать взаимовыгодные экономические отношения субъектов межфирменной интеграции для достижения поставленных целей с учетом воздействия факторов внешней среды.

Особенностью межорганизационного управления в кластерах является то, что объектом управления является деятельность независимых друг от друга взаимодействующих экономических субъектов, каждый из которых в интегрированной структуре выполняет свои функции.

Организационная структура управления кластером представляет собой систему распределение целей между различными управленческими звеньями. В ее составе следует выделить управленческие подразделения, входящих в кластер организаций, внутрисистемные связи, основанные на распределении между ними задач и функций, ответственности, полномочий и прав.

Система управления кластерным объединением находится под влиянием внутренних факторов и выстраивается с учетом внешних условий формирования и функционирования кластера. К внутренним факторам, определяющим характер системы межорганизационного управления кластером следует отнести: выбранную участниками управленческую стратегию и схему распределения управленческих задач, территориальное расположение и масштабы деятельности кластера, виды экономической деятельности участников кластера и их производственно-технологический потенциал [7].

В кластере присутствует сетевая форма межорганизационного управления деятельностью его участников, которая обеспечивает синергетический эффект и устойчивость во внешней среде. Сущность сетевой формы управления заключается в выстраивании прямых связей между всеми участниками совместной деятельности, в переходе к горизонтальной структуре управления, в замене административных отношений контрактными.

Сетевая форма межорганизационного управления основана на использовании в качестве субъекта управления кластером независимой хозяйственной единицы, заменяющей функциональные управленческие структуры независимых участников объединения. Данная форма управления базируется на высоком уровне доверия между участниками кластера, а также на развитии устойчивых, постоянно развивающихся связях между экономическими субъектами.

При проектировании системы управления кластером следует определить необходимое соотношение степени делегирования полномочий и мобильности управления, исходя из задач и характера деятельности участников. Основываясь на уровневом подходе к системе управления, в кластерах следует выделять ее стратегический и тактический уровни.

Тактический уровень управления кластером представляет собой совокупность управленческих структур отдельных участников, каждый из которых самостоятельно выбирает решения и способ их реализации из множества альтернативных вариантов. Стратегический уровень управления должна представлять структура, в которой в качестве объекта управления выступает кластер как целостная организационная система.

В части совершенствования механизма межорганизационного управления при сетевой форме, можно предложить передать полномочия по управлению кластерным объединением на стратегическом уровне Исполнительной дирекции кластера, которая представляет собой специализированную управленческую структуру, со своим аппаратом и функциями, зависящими от возлагаемой на него управленческой нагрузки. К этим функциям следует отнести: координацию участников; прогнозирование, выработку стратегии; финансовое и производственное планирование; контроль выполнения; анализ результатов. В качестве положительных сторон привлечения к управлению кластером специально создаваемой структуры - Исполнительной дирекции, можно отметить снижение 
коммерческого риска при сохранении оптимального уровня мобильности управления, активизацию действия управленческого механизма, так как управление осуществляется на основе индивидуальной работы с участниками объединения.

Развитие системы межорганизационного управления в кластерах должно основываться на определенных принципах.

Принципы межорганизационного управления, по нашему мнению, можно определить как основные исходные положения, общепринятые правила и закономерности, использование которых позволит осуществлять эффективное управление для достижения высоких результатов деятельности межорганизационных объединений на различных стадиях их жизненного цикла.

Многие ученые, исследуя проблемы сетевого взаимодействия организаций, создания кластерных структур, развития интеграционных связей, определяли принципы функционирования межорганизационных отношений и управления ими.

Так, Е.М. Терешин, предлагает выделить три группы принципов межорганизационного взаимодействия в кластерных структурах, а именно: принципы цели, принципы формы и содержания, принципы управления и взаимодействия [8].

Ф.Е. Караевой определены следующие группы принципов кластерных объединений организаций:

- общесистемные принципы управления (научности, системности, комплексности, экономичности, эффективности, синергизма, динамического равновесия, адаптации, мотивации)

$$
\text { - частные }
$$

$$
\text { принципы }
$$$$
\text { кластера }
$$

регионального

$$
\text { характера }
$$

формирования

(принципы объединения, децентрализации, партнерства, приоритета рыночных механизмов формировании кластера, интеграции стратегии социально-экономического развития региона и корпоративной стратегии кластера; соответствия стратегических целей участников целям формируемого регионального промышленного кластера; индивидуальной выгоды, ресурсной обеспеченности; сбалансированности расхода ресурсов субъектов власти и предприятий; максимального использования существующего потенциала) [5].
Н.А. Ефимов и Е.В. Иванова акцентируют внимание на специфических принципах кластерной формы организации:

1) принцип мультиколлинеарности;

2) принцип мультипликативности;

3) принцип наличия центра гравитации [3].

O.B. Несмачных, рассматривает механизм управления кластером, базирующийся на трех уровнях принципов:

1) принципы формирования промышленных кластеров (принцип многомодельности; принцип соблюдения критической массы кластера; принцип сосуществования конкуренции и кооперации; принцип специализации; принцип гибкости границ кластера; принцип диффузии инноваций; принцип соответствия стратегических целей; принцип многоуровневости возникновения синергетического эффекта);

2) принципы оценки стратегической эффективности кластера (принцип максимального охвата; принцип соответствия выбранных критериев оценки целям функционирования кластера; принцип многоэтапности оценки; принцип многокритериальности оценки);

3) принципы стратегического управления кластером (принципы проектного управления и финансирования; эффективного распределения ресурсов; комплексности использования ресурсов; идеологический принцип, принцип структурной оптимизации адаптации, принцип целостности и иерархии) [6].

Э.А. Каменских, сформулированы принципы управления кластером на основе того, что любая система управления состоит из субъекта, объекта и субъектно-объектных связей, и каждой из подсистем соответствуют свои принципы [4].

Проанализировав различные подходы по вопросу принципов создания и функционирования кластерных объединений организаций, предлагаем систематизировать принципы межорганизационного управления (таблица 1). Группировка принципов управления кластерными объединениями произведена нами исходя из укрупненных стадий жизненного цикла организаций и видов управления, выделив:

- принципы управления на стадии формирования кластерных структур;

- принципы управления на стадии функционирования кластерных объединений;

- принципы стратегического управления кластерными объединениями. 
Таблий 1

\section{управления кластерными объединениями организаций}

Table 1

Systematization of the principles of interorganizational management in Clusters

\begin{tabular}{|c|c|}
\hline $\begin{array}{c}\text { Группа } \\
\text { принципов }\end{array}$ & $\begin{array}{c}\text { Перечень принципов } \\
\text { управления }\end{array}$ \\
\hline \multirow{5}{*}{$\begin{array}{c}\text { Принципьл } \\
\text { управления на } \\
\text { стадии } \\
\text { формирования } \\
\text { кластерных } \\
\text { структур }\end{array}$} & 1. Принцип системности \\
\hline & 2. Принцип комплексности \\
\hline & 3. Принцип многомодельности \\
\hline & 4. Принцип синергизма \\
\hline & $\begin{array}{l}\text { 5. Принцип оптимальности } \\
\text { размера и структуры кластера }\end{array}$ \\
\hline \multirow{11}{*}{$\begin{array}{c}\text { Принциипь } \\
\text { управления на } \\
\text { стадии } \\
\text { функичонирования } \\
\text { кластерных } \\
\text { объединений }\end{array}$} & $\begin{array}{l}\text { 6.Принципы организации } \\
\text { систем и процессов }\end{array}$ \\
\hline & 7. Принцип координации \\
\hline & $\begin{array}{l}\text { 8.Принцип прозрачности и } \\
\text { открытости }\end{array}$ \\
\hline & 9. Принцип инновационности \\
\hline & 10. Принцип адаптируемости \\
\hline & $\begin{array}{l}\text { 11.Принцип единой } \\
\text { информационной среды }\end{array}$ \\
\hline & 12. Принцип эффективности \\
\hline & $\begin{array}{l}\text { 13. Принцип } \\
\text { мультипликативности }\end{array}$ \\
\hline & $\begin{array}{l}\text { 14. Принцип кооперации и } \\
\text { конкуренции }\end{array}$ \\
\hline & $\begin{array}{l}\text { 15. Принцип ресурсной } \\
\text { обеспеченности и } \\
\text { сбалансированности }\end{array}$ \\
\hline & $\begin{array}{l}\text { 16. Принцип контроля и } \\
\text { оценки эффективности }\end{array}$ \\
\hline \multirow{4}{*}{$\begin{array}{c}\text { Принциины } \\
\text { стратегического } \\
\text { управления } \\
\text { кластерными } \\
\text { объединениями }\end{array}$} & 17. Принцип плановости \\
\hline & $\begin{array}{l}\text { 18. Принцип целостной } \\
\text { системы управления }\end{array}$ \\
\hline & $\begin{array}{l}\text { 19. Принцип } \\
\text { ориентированности }\end{array}$ \\
\hline & 20. Принцип бенчмаркинга \\
\hline
\end{tabular}

Управление кластерными структурами на стадии их создания должно опираться на ряд взаимосвязанных принципов.

Во-первых, следует придерживаться принципа системности, управление на этапе формирования базируется на включении в состав кластера взаимосвязанных организаций, имеющих непосредственную связь минимум с одним участником кластерного образования.

Далее вступает в действие принцип многомодельности, так как требуется принять управленческое решение, выбрав одну из моделей формирования кластерных структур. В настоящее время существует несколько моделей формирования кластерных объединений организаций: начальноцентрализованная, завершенноцентрализованная и равнораспределенная [2].

Выбор той или иной модели формирования кластера должен базироваться на принципе комплексности, согласно которого управление кластерными структурами предполагает комплексное исследование внешних и внутренних факторов, влияющих на формирование кластера. Среди внешних системообразующих факторов выделяют: природно-климатические, политические, социальные, факторы государственного регулирования, ресурсные и рыночные факторы. Внутренними системообразующими факторами являются конкурентные, инновационные, трудовые и производственные связи [5].

Кластерные образования состоят из определенного количества участников, это представители бизнеса, организации рыночной инфраструктуры, научно-исследовательские и образовательные организации, органы власти, поэтому управляя кластерным объединением организаций на стадии формировании необходимо учитывать принцип оптимальности размера и структуры, нарушение которого может отрицательно сказаться на эффективности деятельности. Оптимальный размер кластерного образования зависит от множества внешних и внутренних факторов, а также от выбранной модели формирования кластера, это свидетельствует о тесной взаимосвязи данного принципа с принципами комплексности и многомодельности.

Управляя кластерными образованиями на стадии их формирования также следует придерживаться принципа синергизма, согласно которого взаимодействие участников кластера для достижения общих целей осуществляется таким образом, что потенциал кластера выше суммы потенциалов его участников [5]. На наш взгляд, соблюдение данного принципа при формировании кластерных объединений организаций является основой успешного функционирования и развития кластера.

Управление на стадии функционирования кластерных объединений должно быть основано на общеизвестных принципах организации систем и процессов таких, как: специализация, пропорциональность, параллельность, прямоточность, непрерывность, ритмичность, принцип технической оснащенности. Среди вышеперичисленных принципов особую значимость имеют принцип прямоточности, обеспечивающий кратчайший путь движения 
ресурсов и информации между субъектами кластера и принцип непрерывности, ориентированный на сокращение перерывов между операциями и процессами при взаимодействии участников кластерного образования. Принцип ритмичности реализуется на основе действия принципов пропорциональности и непрерывности [3].

Работа участников кластера базируется на их скоординированных действиях, поэтому управление деятельностью кластерных структур на стадии их функционирования основывается на принципе координации, участники кластера действуют не опосредованно друг от друга, а находясь в тесной взаимосвязи.

В процессе управления кластерным образованием следует придерживаться принципа ресурсной обеспеченности и сбалансированности, следую которому материальные, трудовые, финансовые, информационные ресурсы должны быть сбалансированы, рационально распределены и эффективно использованы субъектами кластера.

Управляя кластером на стадии его функционирования, следует помнить, что данная межорганизационная структура ориентирована на интенсивный экономический рост, посредством внедрения и распространения инноваций между субъектами кластера, причем необходимо создать условия для обеспечения максимального мультипликативного эффекта, при котором эффект в отдельной отрасли порождает благоприятные предпосылки для возникновения в сопряженных отраслях, в этом находят проявления принципы инновационности и мультипликативности [3].

Управленческие решения принимаются на основе информации, для того, чтобы решения были эффективными должны действовать принцип прозрачности и открытости и принцип единой информационной среды, которые подразумевают наличие достоверной и полной и информации, а также создание к ней свободного доступа субъектами кластера (при соблюдении условий конфиденциальности, если требуется). Для рационального использования времени и принятия своевременных управленческих решение возможно создание единой электронной базы для обмена и хранения информации субъектами кластерных образований.

В силу того что, кластерные образования действуют не обособленно от обстоятельств внешней среды, необходимо владеть управленческими средствами, позволяющими быстро адаптироваться к динамично меняющимся условиям внешней среды, так проявляется значимость принципа адаптируемости для выживания и эффективного функционирования кластера. Игнорирование данного принципа может привести к тому, что цели деятельности кластера не будут реализованы.

Среди принципов управления кластерными структурами особое место занимает принцип кооперации и конкуренции, так является и стимулятором и регулятором отношений между участниками кластера. Концепция соконкуренции была разработана в середине 1990-х годов, её авторами являются Бранденбургер и Нейлбафф. Раньше понятия конкуренция и сотрудничество рассматривались обособлено, в настоящее время успех фирмы часто определяется её способностью одновременно реализовывать как конкурентные стратегии так и стратегии, направленные на сотрудничество [10]. Соконкуренция предполагает временный отказ от соперничества и установление сотрудничества и партнерства с целью извлечения экономических преимуществ в сфере различных разработок.

Стратегическое управление кластерным объединением организаций является значимым инструментом, обеспечивающим его устойчивое развитие. По нашему мнению, стратегическое управление должно базироваться на ряде принципов.

Принцип целостной системы управления предполагает наличие единого органа управления, в состав которого входят представители от каждого субъекта кластерного образования. Реализация данного принципа управления предполагает учет интересов каждого участника кластерного образования, установление более тесных взаимосвязей и повышение уровня открытости в принятии управленческих решений.

Стратегическое управление кластерными структурами, согласно принципу ориентированности, исходит из того, что цели кластера являются главными, и на них должны быть ориентированы цели участников, при несоответствии целей субъектов кластерного образования стратегическим целям кластера их необходимо скорректировать в соответствие с имеющимися возможностями.

Кроме того, стратегическое управление кластерными образованиями и совершенствование деятельности участников кластера осуществляется на основе принципа бенчмаркинга, который предполагает непрерывную оценку продукции, услуг и методов 
работы субъектов кластера, для улучшения деятельности каждого предприятия в отдельности и кластера в целом.

Для успешного развития кластерных структур необхдимо осуществлять стратегическое планирование. В планах в соответствии с ресурсным потенциалом определяются направления развития, формулируются цели и устанавливаются задачи, поэтому значимую роль играет принцип плановости.

Выводы. Таким образом, в системе управления кластерами можно выделить самостоятельные уровни: стратегический и тактический. Каждый уровень должен быть представлен своими управленческими структурами. Стратегический уровень межорганизационного управления в кластере обеспечивает разработку и реализацию стратегии развития интегрированного формирования, организацию сотрудничества; формирование экономической политики; создание единого информационного пространства и др.

Перечисленные функции управления в кластерах должны реализовываться на основе системы принципов межорганизационного управления, которое охватывает стадии жизненного цикла организаций и основывается на видах управления.

На стадии формирования кластерных структур, особое внимание необходимо уделить таким принципам как принцип системности, принцип синергизма и принцип оптимальности размера и структуры кластера. На стадии функционирования наибольшую значимость имеют специфические для кластерных образований принципы, а именно принцип инновационности, мультипликативности, принцип кооперации и конкуренции. В современных условиях важную роль играет качественная и оперативная информация, в связи с этим управление должно базироваться на принципах прозрачности и открытости, а также должен соблюдаться принцип единой информационной среды. Среди принципов стратегического управления, ориентированных на развитие кластерных структур, приоритетными являются принцип целостной системы управления и принцип ориентированности, в них отражены особенности кластерных объединений организаций.

Грамотное использование принципов управления межорганизационными отношениями позволит кластерным образованиям эффективно функционировать, что будет способствовать повышению конкурентоспособности и, как следствие, отраслевому экономическому росту.

\section{1. Вайлунова Литература \\ Ю.Г.}

Развитие межорганизационных связей как инструмента повышения конкурентоспособности бизнес-субъектов // Региональная экономика и управление: электронный научный журнал. 2015. №3(43). URL: http://eeeregion.ru/article/4302/ (дата обращения: 01.02.2016)

2. Ермолаев Д.В. Принципы формирования и управления и промышленными кластерами // Известия Тульского государственного университета. Экономические и юридические науки. 2015. №1-1. C. 31-35.

3. Ефимов Н.А. О принципах организации процесса формирования экономического кластера // Экономика и управление. 2013. №10(107). С. 53-57.

4. Каменских Э.А. Принципы управления кластерами туристско-рекреационных услуг // Управление экономическими системами: электронный научный журнал. 2014. №4(64). URL: http://uecs.ru/uecs64-642014/item/2877-2014-04-23-0822-45 (дата обращения: 01.02.2016)

5. Караева Ф.Е. Формирование, идентификация и управление конкурентоспособностью регионального промышленного кластера: дис. ... док. экон. наук. СПб., 2014. 323 с.

6. Несмачных О.В. Принципы формирования, стратегического управления и оценки эффективности промышленного кластера // Современные проблемы науки и образования. 2014. №2. С. 409.

7. Рассказова А.Н. Кластер как современная форма управления промышленными предприятиями// Актуальные вопросы экономических наук. 2010. №15-2. C. 204-209.

8. Терешин Е.М. Система принципов создания, функционирования и управления в кластерных образованиях // Экономические науки. 2010. №6(67). C. 197-199.

9. Шерешева, М.Ю. Формы сетевого взаимодействия компаний: курс лекций. М.: НИУ Высшая школа экономики, 2010. 339 с.

10. Lado A.G. Competition, Cooperation and the Search for Economic Rents: A Syncretic Model. The Academy of Management Review. 1997. 22 (1). Pp. 110-141.

11. Porter M.E. Russian Competitiveness: Where Do We Stand? Institute for Strategy and Competitiveness. Harvard Business School. U.S. - Russian Investment symposium URL: http://www.hbs.edu/faculty/Publication\%20Files/CAON_ Russia_2003_Harvard_Symposium_11-1303_CK_db483f56-69d6-41ae-8670-2d165312ee66.pdf (дата обращения: 01.02.2016)

12. Uilson, A. Partnership for distribution channels// Marketing Week.1997. №5. Pp. 78-82. 
13. Новое слово в российской экономике. Государство делает ставки на кластеры, «ПРАЙМ» агентство экономической информации, 2006 г. URL: http://1 prime.ru/Politics/20061004/759842688.html (дата обращения: 01.02.16)

$\begin{array}{ll}\text { References } & \\ \text { 1. Vailunova J.G. The Development of }\end{array}$ Interorganizational Communications as a Tool for Increasing the Competitiveness of Business Subjects. Regional Economy and Management: Electronic Scientific Magazine. 2015. №3(43). URL: http://eeeregion.ru/article/4302/ (date of access: February 1, 2016)

2. Yermolayev D.V. Principles of Formation and Management of Industrial Clusters. News of the Tula State University. Economical and Juridical Science. 2015. №11. Pp. 31-35.

3. Yefimov H.A. About the Principles of Organizing the Process of the Economic Cluster Formation. Economy and Management. 2013. №10 (107). Pp. 53-57.

4. Kamenskikh E.A. Principles of Managing the Tourist and Recreational Services. Management of Economic Systems: Electronic Scientific Magazine. 2014. №4(64). URL: http://uecs.ru/uecs64-642014/item/28772014-04-23-08-22-45 (date of access: February 1, 2016).

5. Karayeva F.E. Formation, Identification and Management of the Regional Industrial Cluster Competitiveness: Dis.... Doctor of Economics. St.Petersburg, 2014. 323 p.

6. Nesmachnykh O.V. Principles of Formation, Strategic Management and Estimation of the Industrial
Cluster Efficiency. Modern Problems of Science and Education. 2014. №2. Pp. 409.

7. Rasskazova A.N. Cluster as a Modern Form of Management of the Industrial Enterprises. Vital Questions of Economic Sciences. 2010. №15-2. Pp. 204-209.

8. Tereshin E.M. System of Principles of Creation, Functioning and Management in Cluster Units. Economic Sciences. 2010. №6 (67). Pp. 197-199.

9. Sheresheva M.Y. Forms of the Companies' Network Interaction: a Course of Lectures. Moscow: NIU Vyisshaya shkola ekonomiki, 2010. 339 p.

10. Lado A.G. Competition, Cooperation and the Search for Economic Rents: A Syncretic Model. The Academy of Management Review. 1997. 22 (1). Pp. 110-141.

11. Porter M.E. Russian Competitiveness: Where Do We Stand? Institute for Strategy and Competitiveness. Harvard Business School. U.S. Russian Investment symposium URL: http://www.hbs.edu/faculty/Publication\%20Files/CAON_ Russia_2003_Harvard_Symposium_11-1303_CK_db483f56-69d6-41ae-8670-2d165312ee66.pdf (date of access: February 1, 2016).

12. Uilson, A. Partnership for distribution channels. Marketing Week. 1997. №5. Pp. 78-82.

13. A new word in the Russian economy. The state is betting on clusters, «PRIME» business news Agency, 2006 year

URL: http://1prime.ru/Politics/20061004/759842688.html (date of access: February 1, 2016). 